\title{
Visual and nonvisual information disambiguate surfaces specified by motion parallax
}

\author{
SHEENA ROGERS and BRIAN J. ROGERS \\ University of Oxford, Oxford, England
}

\begin{abstract}
Motion parallax has been shown to be an effective and unambiguous source of information about the structure of three-dimensional (3-D) surfaces, both when an observer makes lateral movements with respect to a stationary surface and when the surface translates with respect to a stationary observer (Rogers \& Graham, 1979). When the same pattern of relative motions among parts of the simulated surface is presented to a stationary observer on an unmoving monitor, the perceived corrugations are unstable with respect to the direction of the peaks and troughs. The lack of ambiguity in the original demonstrations could be due to the presence of (1) non-visual information (proprioceptive and vestibular signals) produced when the observer moves or tracks a moving surface, and/or (2) additional optic flow information available in the whole array. To distinguish between these two possibilities, we measured perceived ambiguity in simulated 3-D surfaces in situations where either nonvisual information or one of four kinds of visual information was present. Both visual and nonvisual information were effective in disambiguating the direction of depth within the simulated surface. Real perspective shape transformations affecting the elements of the display were most effective in disambiguating the display.
\end{abstract}

Motion parallax has been shown to be an effective and unambiguous source of information about the structure of three-dimensional (3-D) surfaces (Rogers \& Graham, 1979). With the use of a technique analogous to the random-dot stereograms devised by Julesz $(1960,1971)$, motion parallax can be studied in isolation from other possible sources of information (such as occlusion) that normally occur when we move. This technique has provided convincing evidence that motion parallax can supply sufficient information for the perception of depth, shape, and relative position of surfaces in depth (Rogers \& Graham, 1979, 1982, 1983; Graham \& Rogers, 1982). The displays simulate a corrugated surface in the frontoparallel plane under parallel projection. Perception is consistent and unambiguous both when an observer makes lateral movements with respect to a stationary surface and when the surface translates with respect to a stationary observer (Rogers \& Graham, 1979). When the same pattern of relative motions among parts of the simulated surface is presented to a stationary observer on an unmoving monitor, the perceived direction, or sign, of the peaks and troughs of the surface is unstable and reversible.

Figure 1 shows the relative displacement of random dots in a display simulating a square-wave corrugation similar to those used by Rogers and Graham (1979). Alternate bands of dots move in opposite directions but at

We would like to thank Hiroshi Ono for helpful discussions during the early stages of this research. Correspondence concerning this article should be addressed to Sheena Rogers, who is now with the Department of Psychology, University of Wisconsin-Madison, 1202 West Johnson Street, Madison, WI 53706. the same velocity. The center band could equally be behind the surround or in front of it, yet in Rogers and Graham's experiment, the order of the surfaces in depth was reported consistently on every trial. This ambiguity is similar to that found in parallel projections, and, in fact, earlier studies reported poor perception of threedimensionality for a parallel projection of a translating surface and the expected ambiguity of perceived depth order in parallel projections of rotating surfaces (Braunstein, 1966; Wallach \& O'Connell, 1953). The absence of perceived ambiguity in Rogers and Graham's (1979) study therefore needs to be explained.

Motion parallax is not inherently ambiguous. More commonly, motion parallax displays use polar projection and present elements moving in the same direction but at different velocities. The angular velocity of parts of a surface or of separate objects in the visual field is inversely proportional to their distance away from the observer. Theoretically, the faster moving elements would be nearer to the observer and the surface should appear rigid, stable, and unambiguous, even in the absence of observer or monitor motion. Early studies were only partially successful at demonstrating that observers do in fact see the depth order predicted from differences in angular velocity (e.g., Gibson, Gibson, Smith, \& Flock, 1959). More recently, researchers have managed to obtain correct judgments on over $90 \%$ of trials under certain conditions (e.g., Braunstein \& Andersen, 1981; Braunstein $\&$ Tittle, 1988).

Given the theoretical ambiguity of the motion field within Rogers and Graham's (1979) displays, some additional factor (or factors) is clearly providing information 
a

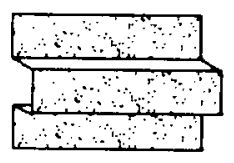

b
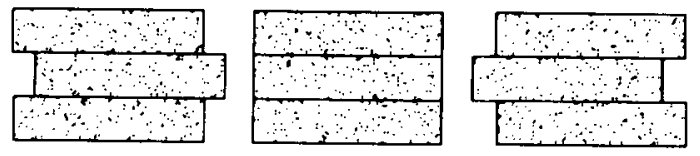

Figure 1. The figure shows (a) the 3-D surface, and (b) the relative displacement of random dots that would be produced by moving the head from left to right. At first, the center band of dots is displaced to the right with respect to the surround, but as the head is moved to the right, the band of dots is progressively displaced to the left. Note that in the actual display, the random dots always completely filled the screen behind a circular aperture so that the edges of the distorting pattern, which could provide additional information, were not visible.

for the depth order of the surfaces. In earlier experiments, perceptual ambiguity with similar displays had been found with the use of both stationary observers and stationary displays. Thus it would seem that for motion parallax information with equal and opposite velocities to be consistent and unambiguous, additional information produced either by the movement of the observer's head, or by the movement of the display relative to the observer's head, is required. The present experiment was an attempt to identify the source of this disambiguating information by systematically controlling potential contributing factors. The absence of ambiguity in Rogers and Graham's original motion parallax displays could have been due to the presence of either (1) nonvisual information produced when the observer or surface moved, or (2) additional optic flow information from the entire array, including objects surrounding the simulated 3-D surfaces. In the present experiment, we examined these alternatives by comparing six different display and viewing conditions. Potential sources of disambiguating information were systematically isolated and tested for their ability to bias perceived direction of depth.

It is possible that the self-produced parallax condition (with head movement) provides important nonvisual information, in the form of proprioceptive and vestibular signals, that is utilized by the visual system in disambiguating the direction of depth in the simulated surface. (In the externally produced parallax condition, such information may also be available from the tracking movements of the eyes and head required during observation of the moving oscilloscope display.) Isolating this nonvisual information allows one to test whether it is sufficient to determine a consistent perceived depth order in a visually ambiguous display. If it is, we would expect that the band of dots moving in a direction opposite that of the observer will appear nearer than the band moving in the same direction.
A second possibility is that additional visual information was available to observers in the Rogers and Graham (1979) experimental paradigm. In both the self-produced and the externally produced parallax conditions, there was an ambiguous pattern of equal and opposite relative motions among the elements of the display itself, defined relative to the monitor. The experiments were not carried out in a completely darkened room, however, and thus the subject could see the frame of the oscilloscope, the layout of the apparatus, and other objects in the room. The dots on the screen also moved in relation to these other objects in the laboratory and in relation to the observer. The complete flow field, or optic array, defined relatively to the observer, is potentially quite different from the monitor-relative velocity field. When this complete observer-relative flow field is considered, additional sources of visual information are available: (1) projective (trapezoidal) changes in the overall shape of the dot pattern, and (2) the pattern of relative motions that exist between the elements of the display and features of the foreground and surrounding surfaces.

Braunstein and Tittle (1988) have identified a third potential source of visual information in the observerrelative array that could disambiguate the depth order of the display. The bands of the display move in opposite directions and at equal velocity only when their motion is defined relative to the monitor. However, in Rogers and Graham's (1979) experiment, either the observer or the monitor was also moving, and so there is an additional common motion component, from the movement of the head or of the oscilloscope, to be added to the velocity field within the display itself. The effect of this is to increase the angular velocity (within the observer-relative flow field) of the band moving in the same direction as the scope, or in the direction opposite that of the observer, and to decrease that of the band moving in the other direction in each case. The resulting flow field no longer has equal and opposite relative motions. All parts of the surface now move in the same direction but at different velocities. This flow field is theoretically unambiguous and Braunstein and Tittle's (1988) results indicate that the velocity field is, indeed, able to govern perceived order in depth.

It is important to separate these different sources of visual information in the observer-relative optic array. In particular, the observer-relative velocity field should remain ambiguous throughout the observation period so that one can examine the contribution of (1) the overall shape changes in the display and (2) the pattern of relative motion between the display and the foreground. This consideration entailed an important change in the original Rogers and Graham (1979) procedure. In a pure translation (of display or observer), all three of the above visual information sources necessarily covary in a natural scene. To allow the overall projective shape of the display to vary independently of the velocity field (maintaining the ambiguous, equal, and opposite pattern), it was necessary to rotate the display about a point underneath the center of the screen in one condition of the present experiment. 
The dot motions on the screen were still translations, but the observer-relative velocity field still consisted of equal velocity motions in opposite directions. Rogers and Graham's procedure actually involved both a large horizontal translation component and a rotation component: In the self-produced parallax condition, the chinrest rotated about a point under the screen, and the head followed a shallow arc path. In the externally produced parallax condition, the oscilloscope pivoted about a point at the back of the scope, and the screen followed a similar shallow arc path. Disambiguation of surface depth order in the present study is therefore highly relevant to the earlier study.

Full descriptions of each of the six display and viewing conditions of the present experiment are given below, along with specific predictions for each condition.

\section{METHOD}

\section{Displays and Apparatus}

The motion parallax displays were generated by the same general method and with similar apparatus as Rogers and Graham's earlier study, described in detail there and elsewhere (Rogers \& Graham, 1979, 1982; Graham \& Rogers, 1982). A computergenerated random-dot pattern was displayed and transformed in real time, exactly simulating the relative displacements among parts of the display that would be produced in the flow field by a real corrugated surface as the observer's head moved from side to side. The pattern was displayed on a large-screen oscilloscope (HP 1304A) positioned $57 \mathrm{~cm}$ from the subject's eye. The screen subtended $20^{\circ}$ (vertically) $\times 25^{\circ}$ (horizontally) of visual angle, but only a circular area subtending $17^{\circ}$ was visible to the observer. The pattern was made up from an array of $256 \times 256$ points, each of which could be illuminated with a $50 \%$ probability. The pattern was generated on a Matrox ALT 256 graphics board interfaced to a Cromenco System 3 computer. All displays simulated a sine-wave corrugation with three complete cycles from top to bottom. The spatial frequency of the 3-D surface was therefore 0.15 cycles per degree (cpd). Maximum relative dot displacement was 50.4 arc min, equivalent to $3.02 \mathrm{~cm}$ of relative depth from the peaks to the troughs of the corrugations. (Note that Rogers \& Graham, 1979, used a smaller screen oscilloscope subtending $12.5^{\circ} \times 10^{\circ}$ of visual angle with a $64 \times 64$ array of points. They set the spatial frequency of the corrugations at $0.1,0.3$, or $0.5 \mathrm{cpd}$ and varied the amplitude of the modulating signal, giving simulated depths from 0.66 to $3.02 \mathrm{~cm}$.)

A system of turntables was constructed to allow independent control of the movement of the oscilloscope and of the foreground. A hardboard and wood viewing tunnel $57 \mathrm{~cm}$ long $\times 60 \mathrm{~cm}$ wide $\times 35 \mathrm{~cm}$ high enclosed the entire foreground area. The oscilloscope was positioned at one end of the tunnel, and a chinrest was fixed to the other end. The tunnel was lined with black card in all except the foreground flow condition, when it was lined with black and white irregularly patterned paper. Either the oscilloscope and the tunnel rested on separate platforms, each of which was $57 \mathrm{~cm}$ long $\times 60 \mathrm{~cm}$ wide, or both objects were placed on one longer platform $114 \mathrm{~cm}$ long $\times 60 \mathrm{~cm}$ wide. The platforms could be rotated to and fro through $15^{\circ}$, pivoting about a point under the center of the oscilloscope screen. The oscilloscope could be raised very slightly and held on metal rods to allow the long platform to be rotated while the oscilloscope itself remained stationary. A circular aperture cut in stiff black card was positioned just in front of the screen and fixed either to the tunnel or to the scope so that it remained stationary during scope or tunnel movement, respectively.
A heavy black card frame $36 \mathrm{~cm}$ high $\times 90 \mathrm{~cm}$ wide surrounded the oscilloscope screen, preventing the observer from seeing any part of the apparatus or the room beyond the display during the experiment.

Viewing was monocular. The random-dot pattern was still, appearing to be 2-D and without structure until either the oscilloscope or the foreground tunnel moved. A potentiometer under the platform monitored its movement. A voltage derived from the potentiometer modulated a sine-wave signal from a Wavetek 175 arbitrary waveform generator that was fed to the $x$-input of the oscilloscope, systematically distorting the random-dot pattern. In the control condition and in conditions with simulated trapezoidal shape transformations, the deforming signal was supplied by a generator and did not depend on movement of the apparatus.

\section{Viewing Conditions}

There were six different viewing conditions: the first was a control condition in which perceived ambiguity was predicted; in the second, nonvisual information for self-motion was isolated; in the third, fourth, and fifth, aspects of overall projective shape changes were respectively examined; and the sixth included a pattern of relative motion in the foreground, between the display and the observer. The essential features of each of these conditions of the experiment are illustrated in Figure 2 (panels a-f). The walls and ceiling of the tunnel are not shown in the figure. The edges of the display always extended beyond the circular aperture and were not visible.

Control condition (Figure 2a). Neither the observer nor the oscilloscope moved, and no other potential source of information was available that could be expected to disambiguate the display. Here, and in all except the foreground flow condition, the entire visible foreground (inside the tunnel) was black and viewing was in dim light to minimize additional optic flow information.

Nonvisual information (Figure 2b). Vestibular and proprioceptive information for egomotion were isolated, and visual information from the surrounding surfaces was controlled. No additional visual information was available to help disambiguate the depth order of parts of the simulated surface. The scope and tunnel were mounted on the long platform, which rotated to and fro with the side-to-side movements of the observer's head. The observer's head was securely held in a chinrest attached to the tunnel. The tunnel was gripped at the sides by the subject, who then moved the entire apparatus smoothly to and fro, making one complete oscillation about every $2 \mathrm{sec}$. The entire array was therefore stationary in relation to the observer, except that the sinusoidal displacement of the pattern of dots within the display was yoked to the movement of the apparatus. If the direction of depth in the surface is perceived consistently here, it follows that vestibular and proprioceptive information can disambiguate motion parallax.

When a frontoparallel, rectangular, planar surface (such as the screen of an oscilloscope) translates or rotates with respect to an observer, the surface projects to a trapezoid in the flow field. This shape change also affects the markings on the surface, and it has three principal components: (1) a linear perspective component in which horizontal lines of elements converge in the array with increasing distance from the observer (since these changes occur along the vertical dimension of the flow field, this component has been called vertical perspective; Braunstein, 1977); (2) a gradient of increasing texture density as the surface recedes, and (3) a horizontal width change. These last two can be called horizontal perspective. Three separate display conditions were employed to investigate the contribution of these projective shape changes.

Real perspective information (trapezoidal shape changes) (Figure 2c). The combined effects of normal perspective were separated from other visual and nonvisual factors. This was achieved without head movement and without oscilloscope translation (and 

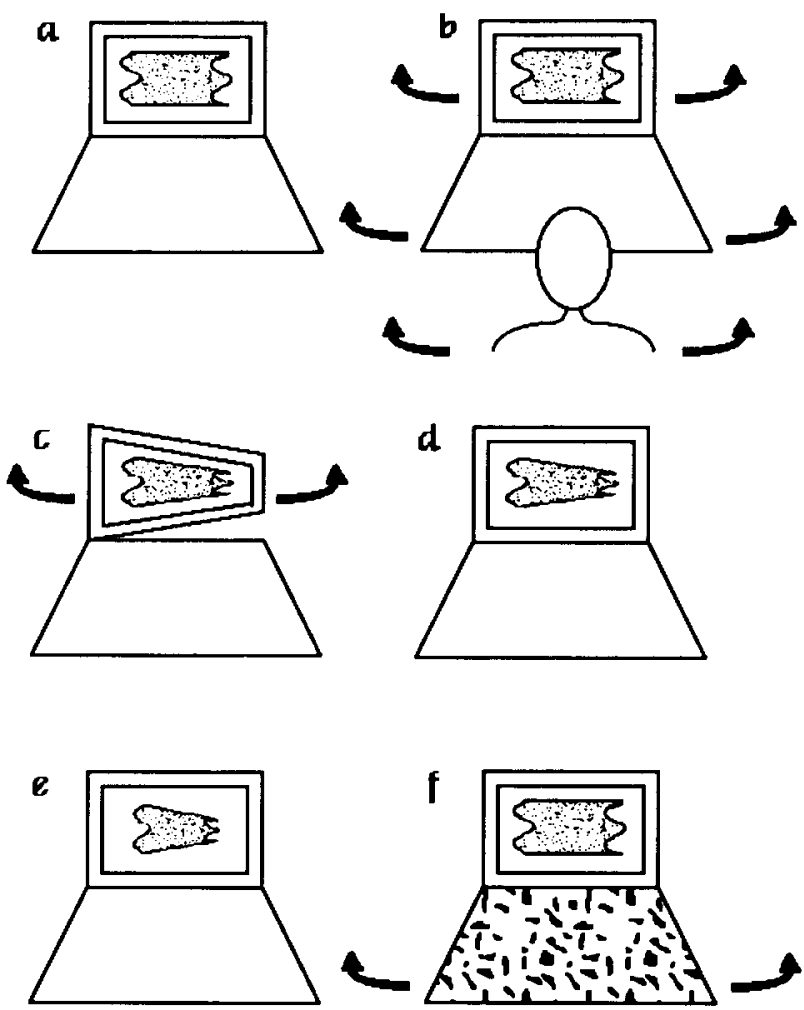

Figure 2. The viewing conditions in each condition of the experiment. Note that, in all cases, the foreground was enclosed in a tunnel. Only the floor is shown here. Arrows indicate movement. (a) Control condition, (b) nonvisual information for egomotion, (c) real perspective transformation, (d) simulated vertical perspective transformation, (e) simulated vertical perspective transformation with compression of elements along the horizontal axis (a "width change"), and (f) foreground now.

a consequent change in the observer-relative velocity field) by rotating the oscilloscope to and fro around a vertical axis passing through the center of the screen. The scope was on the small platform, so the foreground tunnel and the observer were both stationary. The circular aperture was fixed to the scope end of the tunnel, obscuring the transforming contours of the display. The perspective transformations of the display elements provide potentially useful information for the direction of the screen's rotation. If the ability of motion parallax to specify the direction of depth is determined only by the velocity field, then, when the latter is ambiguous, the perceived surface should be ambiguous with respect to its sign. If observers perceive direction consistently, however, differences in angular velocity are not necessary and the presence of additional perspective information is sufficient to disambiguate the surface. The band with dots moving in the same direction as the rotating oscilloscope should appear nearer in this case.

Electronically simulated vertical perspective (trapezoidal shape change) without and with a width change (Figures $2 \mathrm{~d}$ and $2 \mathrm{e}$ ). Braunstein (1977) has shown that normal perspective can disambiguate the direction of rotation of a sphere and that judgments are just as accurate when only vertical perspective information is present. Performance was at chance when only the horizontal component of perspective was included in his display (rotating spheres do not, of course, undergo the additional projective width change of rotating rectangles). Two displays were included in the present experiment in an attempt to separate the effect of vertical perspective from horizontal perspective information. In one of these, the display was deformed electronically to produce a trapezoidal expansion and compression along the $y$-axis of the display, simulating the vertical perspective changes produced by rotating the screen (which remained stationary). The third display added a horizontal width change synchronized with the vertical perspective transformation. The introduced width change compressed the elements of the display uniformly along the horizontal dimension of the display. Texture density did not increase with implied distance from the observer, however. (It was not possible to produce the gradient of texture density appropriate to a slanted surface along the $\boldsymbol{x}$-axis of the display with the present apparatus.) If this width change is necessary information for rotation, its combination with the vertical perspective transformation should provide a more accurate simulation of normal perspective effects, and therefore observer performance should be similar to that in the condition in which the oscilloscope is actually rotated. Unfortunately, it was not possible to simulate these changes accurately, and they were slightly exaggerated. This was expected to cause some perceived elasticity in these displays. Note that the trapezoidal distortions introduced here simulate the same corrugated surface, but in polar, rather than parallel, projection.

Foreground flow (Figure 2f). The sixth display was used to examine the role of the pattern of relative motions among the elements of the display and features of the foreground. The observer and the oscilloscope remained stationary, but a pattern of flow in the foreground was introduced that was equivalent to that produced by a linked movement of the observer and the 3-D object in stationary surroundings, but without the consequent, nonvisual, vestibular and proprioceptive information. The foreground tunnel was mounted on the long platform, but the scope was slightly raised above the platform, preventing it from moving with the foreground. The circular aperture was fixed to the scope. The tunnel was irregularly patterned in black and white on all four interior sides. The room was dimly lit, but a small window in the roof of the tunnel, covered with transluscent perspex, allowed more light to be admitted than in the other conditions. The experimenter rotated the tunnel about the pivot point under the center of the screen at the rate of about one complete oscillation every $2 \mathrm{sec}$. No perspective information in the form of real or simulated trapezoidal shape changes were present in the flow field of this display. The displayspecific relative motions were unaffected, but new relative motions existed between the elements of the display and the elements of the foreground. There was a gradient of angular velocity in the foreground to which motion within the display could be linked. In motion parallax, all objects before the fixation point are displaced in one direction and all objects beyond the fixation point are displaced in the opposite direction. If the information provided by foreground flow is effective, the band in the display with dots moving in the same direction as the foreground elements should reliably appear to be nearer, and the simulated surface should appear to be unambiguous.

\section{Design and Procedure}

Six observers participated in all six conditions of the experiment. Owing to the different apparatus requirements of these conditions, trials were blocked by condition and most subjects completed conditions requiring the long platform (the nonvisual information and foreground flow conditions) in a separate session from that requiring the short platform (the real trapezoidal transformation). The remaining conditions were tested in either session. The order of the blocks was different for each subject. A block consisted of 10 trials, each with a $30-\sec$ duration. The beginning and end of a trial were signaled by a tone. There were a 5 -sec pause between trials and a 5-min rest period between blocks. The initial direction of dot displacement (left or right) in a target band was randomly determined on each trial. (This was achieved by inverting the amplitude 
of the sine-wave signal from positive to negative, or vice versa.) A small spot fixed to the oscilloscope screen marked the target band of the display.

The subject wore an eye patch, and the room lights were dim. Two practice trials were run before each block commenced. The subject's task, when each trial began, was to report whether the target bar was convex or concave (a peak or a trough). Perception of depth order was continuously monitored with a two-button response panel: one button indicated perception of a concave target, and the other, a convex target. Time spent pressing each button was recorded. Subjects were instructed to press neither button when the display did not appear rigid and 3-D. This "not-pressing" time was also recorded throughout the trial. The dependent variable was the percentage of time in each trial during which the direction of the perceived surface was consistent with that predicted for the trial. (No consistent depth order was predicted for the control condition, so data contributing to the higher of the two obtained means on each trial were treated as "consistent" responses for the purposes of the analysis.)

\section{Subjects}

There were 6 subjects. Two graduate students and 2 academic visitors at the Department of Experimental Psychology at the University of Oxford were unfamiliar with motion parallax simulated surfaces and were naive as to the purpose of the experiment. The 2 authors also participated as subjects.

\section{RESULTS}

All subjects reported seeing a compelling, rigid, 3-D corrugated surface and were able to judge a direction in depth in all conditions, on every trial, for most of the $30 \mathrm{sec}$ within any trial $(M=91 \%)$. Typically, subjects would look at the display for a few seconds before pressing either button (accounting for most of the "not 3-D" data in the figure). (Braunstein \& Andersen, 1981, report a similar latency.) In the control condition, in which ambiguity was predicted, observers reported the surface to be rigid and 3-D 88\% of the time, but they experienced several reversals in depth, so that within any trial the target corrugation of the surface appeared to be concave as often as it appeared convex. This is reflected in Figure 3. It can be seen that perception of the sign of the surface was at chance in the control condition (Figure 3a). Perceived depth order was above chance and consistent with the prediction in all remaining conditions, however, and subjects reported that few reversals were experienced (perhaps one or two early in each trial). Perception was most reliable and consistent with the predicted depth order when the display included real trapezoidal transformations. In fact, on most trials, the surface was immediately seen correctly and did not reverse $(M=90 \%$, Figure $3 \mathrm{c})$. Foreground flow and nonvisual information were also effective in disambiguating the surface $(M=75 \%$ and $M=$ $73 \%$; Figures $3 \mathrm{f}$ and $3 \mathrm{~b}$, respectively). Responses to the two simulations of trapezoidal shape transformation were somewhat less consistent, but above chance levels $(M=$ $64 \%$ for the vertical perspective transformation, Figure $3 \mathrm{~d}$; and $M=62 \%$ for the display with a horizontal width change added, Figure 3e). A difference among these six means was supported in a two-factor within-

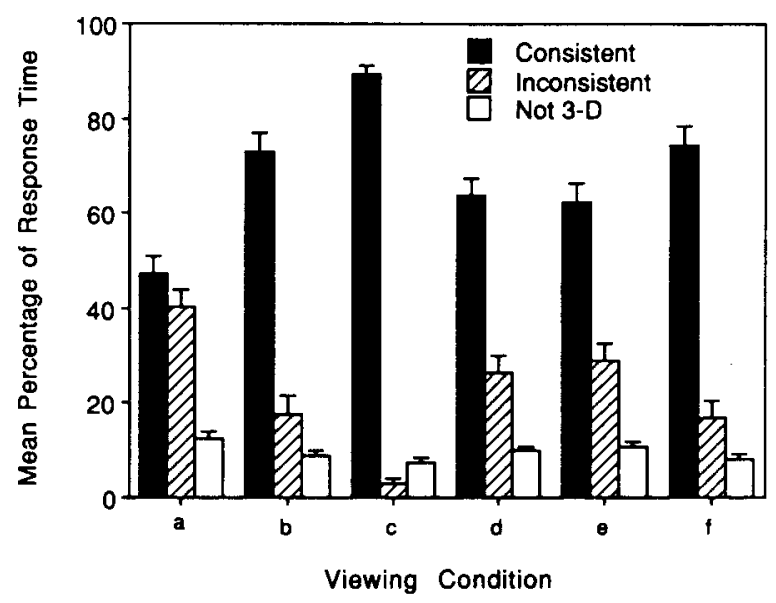

Figure 3. Mean percentage of time per trial during which the perceived depth order of the surface was consistent or not consistent with the predicted order for each viewing condition. (a) Control condition, (b) nonvisual information, (c) real perspective transformation, (d) vertical perspective transformation, (e) vertical perspective transformation plus a width change, and (f) foreground flow. Vertical bars indicate standard error of the mean.

subjects analysis of variance (with viewing condition and repetition number as the factors) $[F(5,25)=7.077, p=$ $.0003]$. No other effects approached significance. Post hoc Tukey $(H S D)$ analyses indicated that the three experimental conditions with the highest means were reliably different from the control condition at $p<.05$ : nonvisual information, real trapezoidal transformations, and the foreground flow condition. The vertical perspective simulation and vertical perspective plus a width change seemed to bias the response somewhat; but these means did not differ from the control condition at conventional levels of significance, and they did not differ from each other $(p>$.05). Both displays were less well disambiguated than the display with real perspective information present $(p<.05)$. No other differences were significant.

The present experiment, unusually, measured the percentage of time on each trial during which the direction of depth in a 3-D surface appeared to be consistent with the theoretical prediction. In earlier studies, subjects gave single reports on the direction of depth in each trial. If observers tend to wait until depth order is stable before reporting their percepts, it is very likely that data from that method would appear even stronger (see, e.g., Rogers \& Graham, 1979, and Braunstein \& Tittle, 1988). We were not able to record observers' final judgments of direction (before each trial ended), which would have given data more closely comparable to earlier measures. However, it is possible to examine the response pattern within each trial and decide whether that trial was mostly consistent with the prediction, mostly inconsistent, or ambiguous, to give percentages of trials correct for each viewing condition. (Each trial lasted $30 \mathrm{sec}$. On the average, for $2.7 \mathrm{sec}$ the display was not rigid and not 3-D. 
From the binomial test, the [one-tailed] probability of observing inconsistent responses for as few as 9 of $27.3 \mathrm{sec}$ is $p<.05$. Hence, trials that met or exceeded this criterion were judged to be consistent with the prediction or, by extension, inconsistent or ambiguous.) Table 1 gives the proportion of trials correct and incorrect by this criterion for each condition, and the relevant means from Figure 3. Performance in the best condition (real trapezoidal transformations) is almost perfect, with just 1 trial in 60 ambiguous (98.3\%), and none incorrect. Foreground flow and nonvisual information are a little less well disambiguated by this measure also $(80 \%$ and $81 \%$, respectively). The two simulations of perspective information were again the least successful ( $73 \%$ and $68.3 \%$ of trials were perceived correctly). For comparison, Rogers and Graham (1979) reported that direction in depth was correctly reported on every trial; Braunstein and Andersen (1981, Experiment 1) obtained $94 \%$ correct with the best ratio of maximum to minimum dot velocity, $73 \%$ with the worst. Braunstein and Tittle (1988) also obtained close to $100 \%$ accuracy in judging depth order with ideal velocity ratios, and $81 \%$ with the least effective ratio. (All of these percentages are for conditions in which ambiguity is not predicted.)

\section{DISCUSSION}

The results of the present experiment once again demonstrate that motion parallax information is sufficient to specify the shape of a 3-D surface. All subjects reported

Table 1

Mean Proportion of Trials and Mean Proportion of Time Per Trial in Which Response is Consistent or Inconsistent With Prediction

\begin{tabular}{lcc}
\hline Viewing Condition & $\begin{array}{c}\text { Proportion } \\
\text { of Trials }\end{array}$ & $\begin{array}{c}\text { Proportion of } \\
\text { Time per Trial }\end{array}$ \\
\hline Control (ambiguous) & .44 & .47 \\
$\quad$ Consistent & .31 & .42 \\
Inconsistent & .25 & .11 \\
$\quad$ Ambiguous/not responding & & \\
Nonvisual information & .81 & .73 \\
$\quad$ Consistent & .04 & .18 \\
Inconsistent & .15 & .09 \\
$\quad$ Ambiguous/not responding & & \\
Real perspective & .98 & .90 \\
$\quad$ Consistent & 0 & .03 \\
Inconsistent & .02 & .07 \\
$\quad$ Ambiguous/not responding & & \\
Simulated vertical perspective & .73 & .64 \\
Consistent & .15 & .26 \\
Inconsistent & .12 & .10 \\
$\quad$ Ambiguous/not responding & & \\
Same + width change & .68 & .63 \\
Consistent & .25 & .29 \\
Inconsistent & .07 & .08 \\
Ambiguous/not responding & & .75 \\
Foreground flow & .80 & .08 \\
$\quad$ Consistent & .02 & \\
Inconsistent & & \\
Ambiguous/not responding & &
\end{tabular}

perceiving a compelling, rigid, corrugated surface while parts of the random-dot display pattern were systematically displaced. The extent to which this surface was also stable and unambiguous with respect to the order of parts of the surface in depth was dependent on the presence of information in addition to the relative displacement of the dots. In the absence of this information, the perceived surface was unstable and reversible in depth. It is apparent in the results that both visual and nonvisual information disambiguate motion parallax displays containing theoretically ambiguous velocity fields: nonvisual information in the form of vestibular and proprioceptive signals for egomotion; visual information in the form of projective shape transformations (polar projection); and optic flow information from surfaces surrounding the display. All of this information was present in the earlier study and could explain the lack of perceived ambiguity there (Rogers \& Graham, 1979).

A combination of vertical and horizontal perspective in the flow field produced by the actual rotation of the oscilloscope screen was the most useful visual information, producing the most stable perceived surface within each trial and almost perfect disambiguation. Only visual information was available in the foreground flow condition, and it, too, successfully disambiguated the direction of depth in the surface corrugations. The information was provided by the relative motions within the whole flow field, foreground, and display, most probably by the available motion perspective (the gradient of relative angular velocity).

The two simulations of the projective transformations undergone by a rotating rectangle indicate that such information can bias the perceived depth order of the simulated surface. It is apparent, however, that some ambiguity remained. A consistent depth order was perceived much of the time, but not reliably above the chance performance obtained in the control condition and significantly less often than in the display that we attempted to simulate: that with real perspective shape transformations. There were a number of reports that these two displays were somewhat elastic and nonrigid at times. Some elasticity might be expected during the periods in which the surface is "inverted" according to the prediction, but it may also result from the limited accuracy of the simulation. However, Braunstein (1977) obtained a similar result. His subjects also perceived some elasticity, and he reports an average of $66 \%$ correct responses with simulated normal perspective and $71 \%$ correct with simulated vertical perspective alone. He found that vertical perspective information disambiguated the direction of rotation (and hence depth) of a sphere as successfully as simulated normal perspective (combined horizontal and vertical components).

Although the present experiment and Braunstein's (1977) displays included different horizontal components of perspective, in neither study was a role found for horizontal perspective in disambiguating direction of depth. However, in the case of our displays, the absence of the horizontal texture density gradient that would usually ac- 
company the rotation of a corrugated surface may be important. Its absence, after all, implies a stationary, frontoparallel surface and contradicts the vertical perspective information given. It is possible that an accurate simulation of this projective effect, alone or with an accurate perspective transformation, would more effectively disambiguate the display.

In conclusion, motion parallax effectively specifies 3-D shape. Perception of a consistent depth order from motion parallax is possible, despite an ambiguous velocity field: both additional visual information and nonvisual, vestibular, and proprioceptive information can disambiguate the direction of depth in a simulated surface. Furthermore, since each of the tested sources of information is sufficient alone, none is a necessary condition for effective motion parallax. The results of the present experiment demonstrate that an inherently ambiguous display of the kind used in earlier studies can be perceptually stable, consistent, and unambiguous when additional visual or nonvisual information is provided.

\section{REFERENCES}

BRaUnstein, M. L. (1966). Sensitivity of the observer to transformations of the visual field. Journal of Experimental Psychology, 72, 683-687.

Braunstein, M. L. (1977). Perceived direction of rotation of simulated three-dimensional patterns. Perception \& Psychophysics, 21, 553-557.
Braunstein, M. L., \& Andersen, G. J. (1981). Velocity gradients and relative depth perception. Perception \& Psychophysics, 29, 145-155.

Braunstein, M. L., \& Tittle, J. S. (1988). The observer-relative velocity field as the basis for effective motion parallax. Journal of Experimental Psychology: Human Perception \& Performance, 14, $582-590$.

Gibson, E. J., Gibson, J. J., Smith, O. W., \& Flock, H. (1959). Motion parallax as a determinant of perceived depth. Journal of $E x$ perimental Psychology, 58, 40-51.

Graham, M., Rogers, B. (1982). Simultaneous and successive contrast effects in the perception of depth from motion parallax and stereoscopic information. Perception, 11, 247-262.

JULESZ, B. (1960). Binocular depth perception of computer generated patterns. Bell System Technology Journal, 39, 1126-1162.

JuLESZ, B. (1971). Foundations of cyclopean perception. Chicago: University of Chicago Press.

Rogers, B. J., \& Graham, M. (1979). Motion parallax as an independent cue for depth perception. Perception, 8, 123-134.

Rogers, B. J., \& Graham, M. (1982). Similarities between motion parallax and stereopsis in human depth perception. Vision Research, 22, 261-270.

Rogers, B. J., \& Graham, M. E. (1983). Anisotropies in the perception of three-dimensional surfaces. Science, 221, 1409-1411.

Wallach, H., O'ConNell, D. N. (1953). The kinetic depth effect. Joumal of Experimental Psychology, 45, 205-217.

(Manuscript received September 27, 1991; revision accepted for publication April 10, 1992.) 